COMUNICAC̣ÃO BREVE

RAVEL SILVA BORGES

RENATA P. MAGALHÃES

ANDRÉ C. CARIBÉ

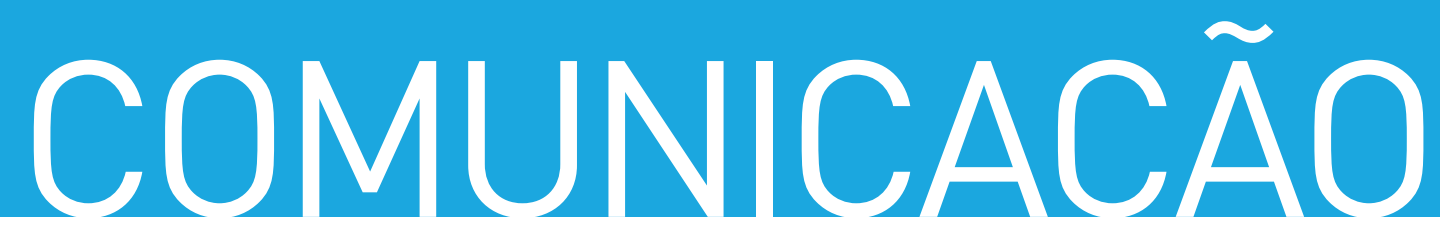

\title{
A TELEPSIQUIATRIA EM UM SERVICCO DOCENTE ASSISTENCIAL DE SAUDE MENTAL: RELATO DE EXPERIÊNCIA, CONSIDERAÇÕES PRÁTICAS E DESAFIOS
}

\section{TELEPSYCHIATRY IN A UNIVERSITY MENTAL HEALTH CARE SERVICE: EXPERIENCE REPORT, PRACTICAL CONSIDERATIONS AND CHALLENGES}

\section{Resumo}

O surto por COVID-19, declarado como uma pandemia pela Organização Mundial de Saúde (OMS) no dia 11 de março de 2020, levou a um colapso no sistema de saúde de vários países em um curto período de tempo. Medidas de isolamento social foram impostas para evitar a rápida propagação do vírus, criando desafios repentinos para a adequada continuidade na prestação dos serviços de saúde mental sem expor profissionais e pacientes ao risco de contaminação. Nesse cenário, a telemedicina desponta como ferramenta fundamental na assistência a portadores de transtornos mentais. $O$ objetivo deste artigo é relatar a experiência de implementação da telepsiquiatria no Ambulatório de Saúde Mental (ASM) da Escola Bahiana de Medicina e Saúde Pública (EBMSP), localizada na cidade de Salvador (BA), assim como discutir os benefícios e dificuldades dessa estratégia.

Palavras-chave: Telemedicina, telepsiquiatria, COVID19.

\section{Abstract}

The outbreak of COVID-19 was declared a pandemic by the World Health Organization (WHO) on March 11, 2020. In a short time, it led the health systems of several countries to collapse. Social isolation measures were imposed to prevent the rapid spread of the virus, creating sudden challenges for the adequate continuity of the mental health care services without exposing professionals and patients to the risk of contamination. In this scenario, telemedicine emerges as an essential tool to ensure assistance to patients with mental disorders. The objective of this article is to report on the experience of telepsychiatry implementation at the mental health outpatient clinic of Escola Bahiana de Medicina e Saúde Pública (EBMSP), located in the city of Salvador, state of Bahia, as well as to discuss the benefits and difficulties of this strategy.

Keywords: Telemedicine, telepsychiatry, COVID-19.

\section{INTRODUÇÃO}

No dia 30 de janeiro de 2020, a Organização Mundial de Saúde (OMS) declarou o surto por COVID-19 (doença causada pelo vírus SARSCoV-2) como uma emergência de saúde pública de importância internacional, tendo sido caracterizada, em 11 de março de 2020, como uma pandemia ${ }^{1}$. A rápida transmissibilidade do vírus entre humanos e a necessidade de hospitalização de doentes que evoluem com a forma mais grave podem levar, em um curto período de tempo, a um colapso no sistema de saúde em diversos países². Para evitar a rápida propagação da doença, medidas rígidas de isolamento social foram implementadas pelas autoridades sanitárias, gerando, assim, uma ruptura abrupta na rotina dos indivíduos e na dinâmica do funcionamento das cidades. Pandemias, inevitavelmente, ocasionam um estado de tensão 
1 Médico psiquiatra. Professor auxiliar, Escola Bahiana de Medicina e Saúde Pública (EBMSP), Salvador, BA. ${ }^{2}$ Acadêmica de Medicina, EBMSP, Salvador, BA. ${ }^{3}$ Mestre e Doutor em Neurociências pela Universidade Federal da Bahia (UFBA), Salvador, BA. Professor adjunto de Psiquiatria, EBMSP, Salvador, BA. Pesquisador, Grupo de Pesquisa em Neurociências, Emoção, Cognição e Comportamento, EBMSP, Salvador, BA.

e ansiedade coletiva. Especificamente em relação à COVID-19, essa tensão é agravada pelo fato de ainda existirem muitas incertezas sobre a fisiopatologia e o adequado tratamento da doença. Essa situação, certamente, impacta a saúde mental das pessoas, pois o isolamento repentino de familiares e amigos, o medo, a ansiedade, o estresse e as alterações nos padrões de sono e alimentação podem desencadear patologias mentais ou agravar condições mórbidas preexistentes ${ }^{3,4}$. Esse cenário criou desafios repentinos para a adequada continuidade na prestação dos serviços de saúde mental, sem expor profissionais e pacientes ao risco de contaminação ${ }^{5}$. Para tentar equacionar essa questão, a telemedicina desponta como ferramenta fundamental na assistência a portadores de transtornos mentais.

O termo telemedicina refere-se ao uso da tecnologia de telecomunicações para a avaliação e o tratamento remotos de pacientes. Embora seja, frequentemente, usado de forma intercambiável com o termo telessaúde, este último, normalmente, indica um escopo mais amplo de serviços de saúde, envolvendo não apenas avaliação e tratamento clínicos, mas também atividades não clínicas, como educação do paciente, treinamento de fornecedores e reuniões administrativas. Telepsiquiatria é o termo dado à aplicação da telemedicina na especialidade da psiquiatria e, normalmente, envolve uma interação entre um psiquiatra e um paciente por


não são novos, porém ganharam grande destaque e importância no contexto da pandemia.

Diante disso, o objetivo deste artigo é relatar a experiência de implementação da telepsiquiatria no Ambulatório de Saúde Mental (ASM) da Escola Bahiana de Medicina e Saúde Pública (EBMSP), localizada na cidade de Salvador (BA), assim como discutir os benefícios e dificuldades dessa estratégia.

O ASM da EBMSP é uma unidade docente assistencial, que atende, exclusivamente, pacientes do Sistema Único de Saúde (SUS), com funcionamento de segunda a sextafeira no período vespertino. Trabalham três psiquiatras assistentes e mais dois psiquiatras para realização de triagem de novos pacientes. Além dos docentes, atuam nesse ambulatório internos do $11^{\circ}$ semestre do curso de medicina, psicólogos e estagiários do curso de psicologia.
Após a confirmação do primeiro caso de coronavírus no estado da Bahia, a coordenação do ASM determinou a suspensão dos atendimentos presenciais, em caráter temporário, para a preservação da saúde da comunidade e dos diversos profissionais que atuam naquele espaço, assim como a suspensão, também temporária, da presença de estudantes. Após a discussão dos detalhes para a reorganização do processo de trabalho e levando em conta as necessidades de cuidados da população, procedeu-se à nova organização, através dos seguintes passos:

1. A equipe de agendamento entrou em contato, por telefone, com todos os pacientes que estavam marcados para atendimento presencial e que tiveram suas consultas canceladas por conta da pandemia, oferecendo a opção de atendimento por telemedicina. Nesse momento, foram passadas informações aos pacientes e familiares acerca das orientações das autoridades sanitárias, no que diz respeito ao distanciamento social e outras medidas de prevenção de contágio. As triagens presenciais para novos usuários foram temporariamente suspensas, assim como as terapias realizadas em grupo.

2. Após concordância dos pacientes, as consultas remotas passaram a ser marcadas de acordo com os dias e horários de atendimento do médico assistente. Antes de conectar-se com o paciente, o médico examinava o prontuário eletrônico e, em seguida, através de um smartphone disponibilizado pela instituição, realizava a teleconsulta por meio de chamada de vídeo do aplicativo WhatsApp. Ao final, receitas e relatórios eram arquivados pela equipe administrativa em local específico, e os pacientes e/ou familiares eram orientados a buscálos no ambulatório, segundo as normas de proteção individual. Nessa mesma ocasião, reagendavamse as consultas de acompanhamento.

3. Como alguns pacientes não dispunham de recursos para serem atendidos via telemedicina, resolveu-se contatá-los através de chamadas telefônicas convencionais. Se, dessa forma, fossem identificadas instabilidades importantes, a família era orientada a levá-lo ao ambulatório para uma consulta presencial, previamente marcada, 
RAVEL SILVA BORGES

RENATA P. MAGALHÃES

ANDRÉ C. CARIBÉ

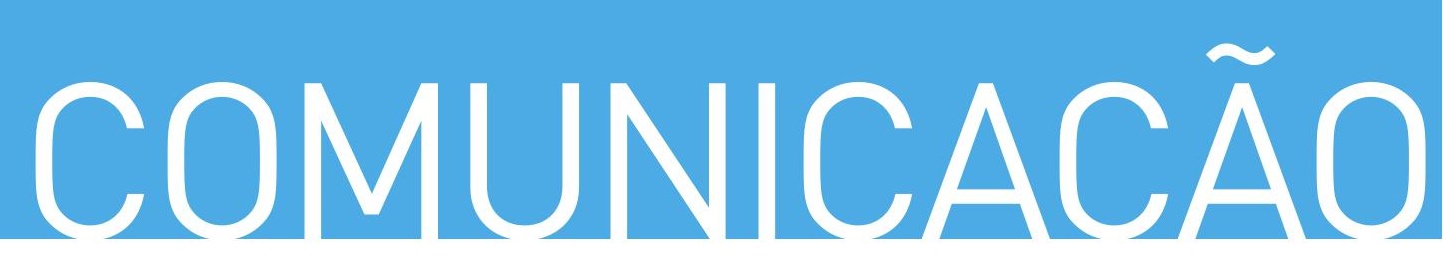

em dia e horários específicos. Caso contrário, apenas renovava-se a receita. Do mesmo modo, quando algum paciente atendido à distância se desestabilizava, manifestando ideação suicida ou heteroagressividade, por exemplo, uma consulta presencial era imediatamente programada ou orientações eram dadas ao paciente e à sua família sobre serviços de emergência disponíveis.

Com essa estratégia relativamente simples, realizamos o atendimento de 236 pacientes de abril a junho de 2020, de um total de 311 pacientes acompanhados em nosso serviço, o que corresponde a uma taxa de 75,8\% de cobertura. Foi também possível manter contato com suas famílias, a fim de orientá-las em relação ao manejo da doença mental, uso de medicações, características da COVID-19 e medidas comportamentais que podem ser adotadas para amenizar os impactos do isolamento social.

Apesar de alguns estudos sinalizarem que o atendimento à distância é similar ao presencial, é necessário ter cautela ao avaliar esses dados iniciais, visto que ainda existem poucas pesquisas nessa área e, na nossa experiência, constatamos algumas dificuldades que valem a pena ser ponderadas ${ }^{7}$. Na nossa realidade socioeconômica, muitos pacientes vivem em casas pequenas ou cômodos, onde não há a privacidade adequada, dificultando a abordagem de determinados assuntos. Alguns dos nossos pacientes se mostraram constrangidos em falar sobre os seus sintomas ou sobre a dinâmica familiar, com receio de que outras pessoas presentes na casa escutassem. Nós decidimos utilizar métodos simples de comunicação virtual, como a chamada de vídeo do WhatsApp, na tentativa de minimizar as dificuldades de manejo tecnológico que previmos por conta do perfil de nossos pacientes, que são, em sua maioria, de baixa renda e baixa escolaridade. Mesmo assim, em poucos momentos, alguns deles, principalmente os mais idosos ou aqueles com comprometimento cognitivo, acidentalmente se desconectavam ou desligavam a câmera ou microfone. O aplicativo WhatsApp pode ser menos seguro do que uma plataforma específica para a realização de consultas, porém se mostrou mais prático e eficaz para o perfil de nossa população, como constatado em outros serviços de psiquiatria ${ }^{8}$. Algumas famílias também não dispunham de equipamento adequado ou tinham acesso à internet de má qualidade, ocorrendo constantes travamentos e quedas de conexão. Outro ponto muito relevante é que, embora na maioria dos atendimentos em psiquiatria não seja necessária a realização do exame físico, perdemos, no atendimento remoto, muitos detalhes do exame psicopatológico desses indivíduos. A forma como chegam ao consultório, como estão vestidos, se exalam algum odor, se estão conversando ou não na recepção, a forma que nos cumprimentam, o jeito de andar, sentar, gesticular, as sutis alterações da mímica facial, o olhar, o tom de voz e até a naturalidade da fala e a prosódia, de certa forma, ficam comprometidos com o atendimento através de telas. Apesar dessas dificuldades, o recurso da telepsiquiatria foi fundamental para manter a estabilidade, o tratamento e minimizar os impactos do distanciamento social nos nossos pacientes. Estes, em sua maioria, reconheceram o suporte como essencial e se sentiram acolhidos e amparados.

A telepsiquiatria se mostrou uma solução muito eficaz para equacionar o distanciamento social e a necessidade de não interrupção dos atendimentos impostos pela pandemia. Apesar de sua utilização já vir sendo feita há muitos anos, a situação crítica atual acelerou sua implementação e ratificou sua necessidade como parte do cuidado ao doente mental. Nos próximos anos, espera-se que esta área seja um campo fértil de pesquisa e que as faculdades de medicina incorporem aos seus currículos o treinamento dos estudantes em telemedicina, tendo a sensibilidade de levar em conta as características socioeconômicas e cognitivas da população a ser atendida. Para que isso ocorra de maneira homogênea e estruturada, é fundamental a revisão das leis e normas brasileiras sobre a regulamentação do atendimento remoto.

Artigo submetido em 29/07/2020, aceito em 16/09/2020. Os autores informam não haver conflitos de interesse associados à publicação deste artigo.

Fontes de financiamento inexistentes.

Correspondência: Ravel Silva Borges, Escola Bahiana de Medicina e Saúde Pública. Av. Dom João VI, 275, Brotas, CEP 40290-000, Salvador, BA. Tel: +55 (71) 3276-8200. E-mail: ravelsborges@bahiana.edu.br 
1 Médico psiquiatra. Professor auxiliar, Escola Bahiana de Medicina e Saúde Pública (EBMSP), Salvador, BA. ${ }^{2}$ Acadêmica de Medicina, EBMSP, Salvador, BA. ${ }^{3}$ Mestre e Doutor em Neurociências pela Universidade Federal da Bahia (UFBA), Salvador, BA. Professor adjunto de Psiquiatria, EBMSP, Salvador, BA. Pesquisador, Grupo de Pesquisa em Neurociências, Emoção, Cognição e Comportamento, EBMSP, Salvador, BA.

\section{Referências}

1. Organização Mundial deSaúde(OMS), Organização Pan-Americana de Saúde (OPAS). OMS declara emergência de saúde pública de importância internacional em relação a novo coronavírus [Internet]. 2020 Jan 30 [cited 2020 May 26]. www.paho.org/bra/index.php?option=com content\&view =article \&id=6100:omsdeclara-emergencia-de-saude-publica-deimportancia-internacional-em-relacao-a-novocoronavirus\&Itemid=812

2. Sujita Kumar Kar, Yasir Arafat SM, Kabir R, Sharma P, Saxena SK. Coping with mental health challenges during COVID-19. In: Saxena S, editors. Coronavirus disease 2019 (COVID-19). medical virology: from pathogenesis to disease control. Singapore: Springer; 2020. p. 199-213.

3. da Silva AG, Miranda DM, Diaz AP, Telles AL, Malloy-Diniz LF, Palha AP. Mental health: why it still matters in the midst of a pandemic. Braz J Psychiatry. 2020;42:229-31.

4. Rajkumar RP. COVID-19 and mental health: a review of the existing literature. Asian J Psychiatr. 2020;52:102066.

5. Ho CS, Chee CY, Ho RC. Mental health strategies to combat the psychological impact of COVID19 beyond paranoia and panic. Ann Acad Med Singap. 2020;49:155-60.

6. O'Brien M, McNicholas F. The use of telepsychiatry during COVID-19 and beyond. Ir J Psychol Med. 2020 May 21;1-6. doi: 10.1017/ipm.2020.54. Online ahead of print.

7. Seritan AL, Heiry M, Iosif AM, Dodge M, Ostrem $J$ L. Telepsychiatry for patients with movement disorders: a feasibility and patient satisfaction study. J Clin Mov Disord. 2019;6:1.

8. Fagiolini A, Cuomo A, Frank E. COVID-19 diary from a psychiatry department in Italy. J Clin Psychiatry. 2020;81:20com13357. 